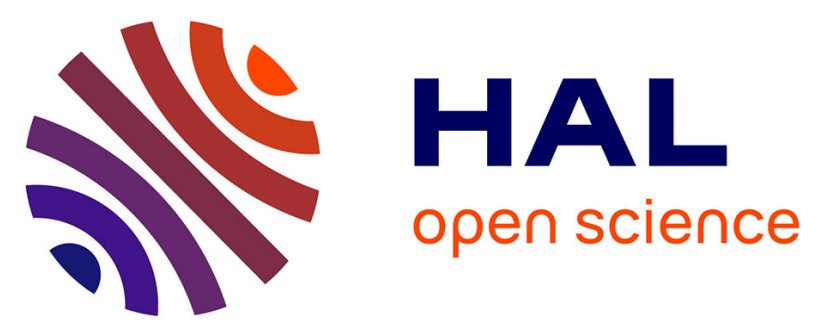

\title{
New constitutive modeling for time-dependent mechanical behavior of polymers close to glass transition: Fundamentals and experimental validation
} Noëlle Billon

\section{- To cite this version:}

Noëlle Billon. New constitutive modeling for time-dependent mechanical behavior of polymers close to glass transition: Fundamentals and experimental validation. Journal of Applied Polymer Science, 2012, Contributions from the 4th International Conference on Polymer Behavior (IUPAC), Lodz, Poland, September 19-23, 2010, 125 (6), pp.4390-4411. 10.1002/app.36598 . hal-00682763

HAL Id: hal-00682763

https://hal-mines-paristech.archives-ouvertes.fr/hal-00682763

Submitted on 23 Aug 2017

HAL is a multi-disciplinary open access archive for the deposit and dissemination of scientific research documents, whether they are published or not. The documents may come from teaching and research institutions in France or abroad, or from public or private research centers.
L'archive ouverte pluridisciplinaire $\mathbf{H A L}$, est destinée au dépôt et à la diffusion de documents scientifiques de niveau recherche, publiés ou non, émanant des établissements d'enseignement et de recherche français ou étrangers, des laboratoires publics ou privés. 


\title{
New Constitutive Modeling for Time-Dependent Mechanical Behavior of Polymers Close to Glass Transition: Fundamentals and Experimental Validation
}

\author{
Noëlle Billon \\ Mines Paris Tech, Ecole Nationale Supérieure des Mines de Paris, CEMEF, UMR 7635, BP 207, 06904 Sophia \\ Antipolis cedex, France
}

Correspondence to: N. Billon (noelle.billon@minesparistech.fr).

Contract grant sponsor: The European Commission, the Framework 6 Program, the Apt-Pack strep project; contract grant number: STREP 505204-1.

\begin{abstract}
The 1D limit of a 3D visco-hyperelastic model is discussed and validated using a rich and rigorous database obtained on PMMA above $T_{g}$. Inelastic phenomena are accounted for as an evolution of internal variables assumed to be related to the alteration of microstructure that induces changes in well chosen constitutive parameters and dissipation of energy. Inelastic strain-rate is deduced from energy balance between elastic and dissipative phenomenon. The concept allows introducing time effects in any rubber elasticity theory, resulting in a viscohyperelastic model consistent with continuum thermodynamics. In this article, Edwards-Vilgis' model is used as the core and extended to a general time dependent constitutive model. Phenomenological models for disentangle-
\end{abstract}

\section{INTRODUCTION}

Modeling of the mechanical behavior of polymers is still a nontrivial task despite of many proposals in the literature. Intrinsic difficulties are the nonlinearity of behavior, even at moderate strain, and the combination of viscoelastic, viscoplastic, strain-hardening, and damaging processes without any clear thresholds in between. Additionally, high sensitivity to temperature ruled by relaxations makes it difficult proposing a general model.

Putting micromechanics models aside, approaches in the literature are often based on a standard modeling (standard materials) that decomposes any experimental observation into three elementary components that are either reversible-instantaneous (named elastic part), or irreversible-not dependent upon strain-rate (named plastic part) or irreversible-dependent upon strain-rate (named viscous part), respectively. Those three contributions to global ment or crystallization are suggested that allow reproducing all viscoelastic effects using one unique model. An accounting for temperature and strain-rate effects by an "a priori" uses of time temperature superposition principle is also proposed. The three concepts result in an original model of high efficiency. This new approach is a highly attractive way of modeling time dependent behavior of polymers over a wide range of temperature and strain-rate up to large strains. This is demonstrated in the case of PMMA.

Key words: time-dependent behavior; large-strain; polymers; inelasticity; PMMA

behavior are combined, either in a parallel, or in a serial manner, assuming that the global stress or, respectively, the global strain, results from the addition of the elementary ones (resp. stresses or strains associated to each of the component). In the frame of thermodynamics, this corresponds to the assumption that global strain is (parallel models) or is not (serial models) an internal variable, being some (parallel models) or all (serial models) the individual strains other internal variables that rule the energy of the system.

To suggest mathematical writings, authors generally use two types of approaches:

- One route is the building up of a 1D-model usually depicted by schematics combining springs and dashpots. In such a case, generating a 3D model is an additional task to be performed carefully at a mathematical level.

- Second route are models, consistent with continuum thermodynamics, ${ }^{1,2}$ in which global behavior is ruled by energy potentials and dissipation pseudo-potentials that depend upon chosen internal variables and parameters.

Whatever the route is, basic assumptions are equivalent in the most rigorous studies, despite of some mathematical differences. 
Consequently, parameters of macroscopic models are the elastic parameters of springs, viscosities of dashpots and, eventually, parameters related to plastic strain (yield, flow rule, see in example $e^{3-5}$ ). Any of those elements can be chosen linear or not linear with respect to the strain and/or the strain-rate, depending on the targeted level of complexity.

As far as polymers are concerned, nonlinearity can be addressed at two levels:

- However, important strain hardening observed at high strain is often reproduced introducing hyperelastic reversible elements. Apart from the well known 3 or 8-chains and "full networks" statistical proposals, ${ }^{6-8}$ which were recently intensively used (see in example ${ }^{9}$ ), some alternative solutions exist. In a non exhaustive manner, let's only quote the route chosen in this study, which seems to be as efficient ${ }^{10}$ as $n$-chains model. In that case, hyperelastic component is based on a physical approach, first proposed by Ball et al. ${ }^{11}$ and extended by Edwards and Vilgis $^{12}$ to the case of non-Gaussian statistics. This model, which is referred to as EV's approach in this article, considers a polymer network in which entanglements introduce some additional entropic effects. Chains are assumed to have a limited extensibility (that is, this is a non-Gaussian approach). Sweeney et al., ${ }^{13-15}$ in parallel to Buckley et al. ${ }^{16-18}$ and further Gorlier et al. ${ }^{19}$ used this model as a component of a standard material. However, EV's potential allows reproducing most of the shapes of experimental tensile curves $^{20}$ and could be used in another manner.

- However, nonlinear viscous effects can be introduces through "non-Newtonian pseudo plastic, Eyring, or Carreau"-like elements. However, some other modeling has been suggested to account for interaction between viscous disentanglement and elastic recovery of the chains. This was done through the definition of a specific representative volume element ${ }^{21}$ or through the existence of a transient network in amorphous $_{\text {phase. }}{ }^{22-26}$

Nevertheless, those models generally use the same standard global modeling, describing behavior as the arbitrary combination of successive phenomena.

Additionally to nonlinearity, parameters are also assumed to depend upon temperature to account for that important characteristic in polymers behavior (in example ${ }^{27,28}$ ). This general approach results in numerous parameters that are not that easy to identify without specific protocol that preallocates one element or another to one macroscopic phenomenon.

Rare are the attempts to introduce time effects in a more physical manner despite of the fact that it is possible to combine the general rubber hyper elastic theory and some evolution of internal variables, potentially induced by microstructure alteration, ${ }^{29,30}$ during loading, which induces time effects in the constitutive model.

This article deals at illustrating concepts of such a route. General goal is to model visco-hyperelasticity of polymers above $T_{g}$ without arbitrary decomposition into "viscous" and "elastic" stresses or strains. Therefore, a hyperelastic model is extended to account for inelastic processes. Those latter are assumed to result in a kinetics of variation of internal variables that have to be accounted for in the energy balance at any time and induce time effects in the writing.

Model is validated in its 1D form using experimental observations on PMMA above but close to $T_{g}$ as initial model should be valid in that domain. However, it can be extended in a much more general 3D form, consistent with the large strain mechanical approach and with continuum thermodynamics. ${ }^{31}$ Entire mathematical derivation will not be addressed here as main purpose of this article is discussing the physical basements and demonstrating the efficiency of the model.

\section{THEORETICAL DEVELOPMENT}

\section{Introduction: hyperelastic behavior}

From a philosophical point of view model is developed starting from two fundamental ideas that are:

- Dependence of behavior upon strain-rate in materials is not the cause of the viscoelasticity at a physical level. In fact, it is the consequence of the fact that physical processes (i.e., changes in microstructure) involved during deformation depend upon time through an intrinsic kinetics. Therefore, effort to include kinetics of processes in models should results in more relevant theory than those assuming an arbitrary dependence of stress (or energy) upon strain-rate.

- From an experimental point of view, the apparent dependence upon strain-rate and the dependence upon temperature are linked. This linkage is well illustrated as the time-temperature equivalence principle. This principle is generally accepted at low strain (i.e., in the linear viscoelastic domain). However, one sometimes observes that it can, in a phenomenological manner, be extended to high strains. This will be illustrated in this article using experimental data on PMMA. However, authors obtained same results in the case of HIPS, PU, PET, and even PA6-6. In such a case, equivalent strainrate at reference temperature (e.g., as defined in 
a WLF's approach) could be used as a parameter that depicts dependence upon strain-rate and upon temperature as well as their coupled effects. We took such opportunity to simplify equations when model did not totally succeed in developing previous item.

Let us assume that above glass transition temperature any polymer can mainly be modeled as an equivalent entangled network. In that sense, core of the behavior is a hyperelastic body whose energy is ruled by a strain energy density, $W$.

As a first approach, let us consider an incompressible material, being initially isotropic. It can be emphasized that incompressibility assumption could be released in a further version of the model. Anisotropy, for its part, is much more difficult to consider.

Nevertheless, in the case of isotropic and incompressible polymer the principal stresses, $\sigma_{i}(i=1,2$, and 3$)$, are related to the principal elastic extension ratios, $\lambda_{i}^{e}$, through eq. (1).

$$
\sigma_{i}=\lambda_{i}^{e} \frac{\partial W}{\partial \lambda_{i}^{e}}-P \quad(i=1,2,3)
$$

where $P$ is an arbitrary pressure that is introduced due to incompressibility. Boundary conditions allow calculating $P$ and, therefore, defining principal stresses. As two examples, uniaxial tension along 1axis and loading conditions that create a plane-stress field in the (1, 2)-plane, lead to eq. (2) and to eq. (3), respectively.

$$
\begin{gathered}
\sigma_{1}=\lambda_{1}^{e} \frac{\partial W}{\partial \lambda_{1}^{e}} \quad, \sigma_{2}=0, \sigma_{3}=0 \\
\sigma_{i}=\lambda_{i}^{e} \frac{\partial W}{\partial \lambda_{i}^{e}}-\lambda_{3}^{e} \frac{\partial W}{\partial \lambda_{3}^{e}} \quad(i=1,2), \sigma_{3}=0
\end{gathered}
$$

To be complete, $W$-potential depends on internal variables, among which temperature, $T$, some variables that should reflect the microstructure of the material, called $m_{1}, m_{2}, \ldots, m_{n}$ in this article and elastic extension ratios, $\lambda_{i}^{e}$. Concerning those latter, another option exists that consists in assuming that internal variables that represents strain field can be reduced in the three elastic invariants, $I_{1}^{e}, I_{2}^{e}$, and $I_{3}^{e}$ [eq. (4)].

$$
\left\{\begin{array}{l}
I_{1}^{e}=\lambda_{1}^{e^{2}}+\lambda_{2}^{e^{2}}+\lambda_{3}^{e^{2}} \\
I_{2}^{e}=\lambda_{1}^{e^{2}} \lambda_{2}^{e^{2}}+\lambda_{1}^{e^{2}} \lambda_{3}^{e^{2}}+\lambda_{2}^{e^{2}} \lambda_{3}^{e^{2}} \\
I_{3}^{e}=\lambda_{1}^{e^{2}} \lambda_{2}^{e^{2}} \lambda_{3}^{e^{2}}=1
\end{array}\right.
$$

One has to notice that the third invariant, $\mathrm{I}_{3}^{e}$, is equal to 1 in case of incompressible materials. Consequently, eq. (1) can be written as eq. (5).

$$
\begin{aligned}
& \left\{\begin{array}{l}
\sigma_{i}=\lambda_{i}^{e} \frac{\partial W\left(T, \lambda_{1}^{e}, \lambda_{2}^{e}, \lambda_{3}^{e}, \mathrm{~m}_{1}, m_{2}, \ldots, m_{n}\right)}{\partial \lambda_{i}^{e}}-P \text { or } \\
\lambda_{1}^{e} \lambda_{2}^{e}, \lambda_{3}^{e}=1
\end{array}\right. \\
& \left\{\begin{array}{l}
\sigma_{i}=\lambda_{i}^{e} \frac{\partial W\left(T, I_{1}^{e}, e_{2}^{e}, \mathrm{~m}_{1}, m_{2}, \ldots, m_{n}\right)}{\partial \lambda_{i}^{e}}-P \\
I_{3}^{e}=1
\end{array}\right.
\end{aligned}
$$

\section{Time-dependent constitutive behavior}

During loading, microstructure of the material can vary. Let us imagine disentanglement, crystallization, crystalline reorganization, and so on. Whatever those changes are, associated $m$-variables should vary, which represents a potential change in the energy density of the material that has to be compensated for thermodynamics principles to be obeyed.

In consequence, at any time, $t$, the continuous change in the characteristics of the equivalent network, due to the alteration of the microstructure under loading, must be compensated. This is achieved partly by some local elastic recovery in the network, $\frac{\partial \lambda_{i}^{\text {re }}}{\partial t}$. This is the source of inelastic strain in our model, that is, the fraction of elastic extension that has to be released to compensate change in energy due to microstructure processes. Then, in a general manner, at any time one has to consider that:

$$
\sum_{j=1}^{n} \frac{\partial W}{\partial m_{j}} \frac{\partial m_{j}}{\partial t}+\sum_{i=1}^{3} \frac{\partial W}{\partial \lambda_{i}^{e}} \frac{\partial \lambda_{i}^{\text {re }}}{\partial t} \leq 0
$$

where $\partial m_{j} / \partial t$ are the kinetics of the evolution of variable $m_{j}$. Equation (6) can be rewritten as eq. (7).

$$
\sum_{j=1}^{n} \frac{\partial W}{\partial m_{j}} \frac{\partial m_{j}}{\partial t}=-\frac{1}{\beta^{\prime}} \sum_{i=1}^{3} \frac{\partial W}{\partial \lambda_{i}^{e}} \frac{\partial \lambda_{i}^{\text {re }}}{\partial t} \quad \text { with } \quad \beta^{\prime} \geq 1
$$

The additional energy, which causes the existence of $\beta^{\prime}$-parameter, could be dissipated into heat (thermomechanical coupling) or stored via some permanent changes in the microstructure (plastic-like phenomenon). As assumed above, the elastic energy, which is released to make alteration of microstructure possible, is the source of inelastic extension ratios, named $\lambda_{i}^{a}$ in this article [eq. (6)].

To express this latter, let us consider a material under loading. A time $t$, elastic strain results in elastic extension ratios, $\lambda_{i}^{e}$. During further time step, $d t$, the global extensions vary from $\lambda_{i}$ to $\lambda_{i}+d \lambda_{i}$. Actual increase in elastic extensions during $d t$, which controls the increase in stress, results from the combination of this external loading and the internal relaxation. If one decomposes this step into two virtual substeps, which is always acceptable for elastic bodies:

- First, a change in elastic extensions from $\lambda_{i}^{e}$ to $\lambda_{i}^{e}$ $+d \lambda_{i}$, keeping inelastic components constant. 
- Second, the activation of inelastic processes that take place [according to eq. (7)] at constant global extension.

In this specific latter substep, assuming that principal elastic extension ratios and principal inelastic extension ratios are collinear it can be written that:

$$
\frac{\partial \lambda_{i}}{\partial t}=\lambda_{i}^{e} \frac{\partial \lambda_{i}^{a}}{\partial t}+\lambda_{i}^{a} \frac{\partial \lambda_{i}^{\mathrm{re}}}{\partial t}=0 \quad \Leftrightarrow \frac{\partial \lambda_{i}^{\mathrm{re}}}{\partial t}=-\frac{\lambda_{i}^{e}}{\lambda_{i}^{a}} \frac{\partial \lambda_{i}^{a}}{\partial t}
$$

In other words:

$$
\begin{aligned}
& \sum_{j=1}^{n} \frac{\partial \mathrm{W}}{\partial \mathrm{m}_{\mathrm{j}}} \frac{\partial \mathrm{m}_{\mathrm{j}}}{\partial \mathrm{t}}=\frac{1}{\beta^{\prime}} \sum_{\mathrm{i}=1}^{3} \frac{\lambda_{\mathrm{i}}^{\mathrm{e}}}{\lambda_{\mathrm{i}}^{\mathrm{a}}} \frac{\partial \mathrm{W}}{\partial \lambda_{\mathrm{i}}^{\mathrm{e}}} \frac{\partial \lambda_{\mathrm{i}}^{\mathrm{a}}}{\partial \mathrm{t}}=\frac{1}{\beta^{\prime}} \sum_{\mathrm{i}=1}^{3} \sigma_{\mathrm{i}} \frac{\partial \ln \lambda_{\mathrm{i}}^{\mathrm{a}}}{\partial \mathrm{t}} \\
& \text { with } \quad \beta^{\prime} \geq 1
\end{aligned}
$$

Model was derived totally in its $3 \mathrm{D}$ form ${ }^{31}$ but we will restrict this article to the simple case of uniaxial loading, namely $\lambda_{1}=\lambda, \lambda_{2}=\lambda_{3}=1 / \sqrt{\lambda}, \sigma_{1}=\sigma$ and $\sigma_{2}=\sigma_{3}=0$. In this case, eq. (9) is reduced to eq. (10):

$$
\sum_{j=1}^{n} \frac{\partial W}{\partial m_{j}} \frac{\partial m_{j}}{\partial t}=\frac{1}{\beta^{\prime}} \sigma \frac{\partial \varepsilon^{a}}{\partial t} \quad \text { with } \quad \beta^{\prime} \geq 1
$$

where $\frac{\partial \varepsilon^{a}}{\partial t}$ is the so-called inelastic strain-rate.

At this stage, the inelastic strain-rate in the material is known as a function of the rate of alteration of microstructure (more rigorously of the rate of variation of the internal variables associated to microstructure elements).

Finally, to complete the model, the mathematical form for $W$ as well as the $m$-variables together with their kinetics of alteration must be defined.

\section{Physical model for microstructure alteration}

Due to its proved efficiency, ${ }^{13-19}$ we chose EV's model as primary network. This model is based on a statistical approach. Chains are assumed to have a finite extensibility. This constrain is ultimately controlled by one positive parameter $\alpha$, being 0 in the case of a Gaussian chain. Chains are linked by permanent nodes, named crosslinks, of density $N_{c}$ per unit volume. In addition to these latter, entanglements exist that increase the entropy of the material. These latter are introduced as slip links whose density is $N_{s}$ per unit volume. A positive "slipperiness factor", $\eta$, is ultimately defined and related do the degree of mobility of slip links, that is, the distance along which a chain can slip in the slip link. Let us only remind that a zero $\eta$-value correspond to permanent nodes. Finally, some theoretical development about topological constrains suggests that at equilibrium, $\eta$-value should be close to $0.2343 .^{11}$
This model is equivalent to simple Gaussian chain model when $\eta$ and $\alpha$ are 0 . General form for EV's equation is given in eq. (11).

$$
\begin{aligned}
W=\frac{N_{s}^{\sim}}{2} & \sum_{i}\left[\frac{\lambda_{i}^{e^{2}}(1+\eta)\left(1-\alpha^{2}\right)}{\left(1+\eta \lambda_{i}^{e^{2}}\right)\left(1-\alpha^{2} I_{1}^{e}\right)}+\ln \left(1+\eta \lambda_{i}^{e^{2}}\right)\right] \\
+\ln & \left.\left.1-\alpha^{2} I_{1}^{e}\right)\right)+\frac{N_{c}^{\sim}}{2} \\
& \left.\times \sum_{i} \frac{\lambda_{i}^{e^{2}}\left(1-\alpha^{2}\right)}{\left(1-\alpha^{2} I_{1}^{e}\right)}+\ln \left(1-\alpha^{2} I_{1}^{e}\right)\right)
\end{aligned}
$$

where $N_{s}^{\sim}$ and $N_{c}^{\sim}$ are $N_{s} k T$ and $N_{c} k T$, respectively, with $k$ the Boltzmann's constant and $T$, the absolute temperature. It is easily demonstrated that eq. (11) can be rewritten as a function of the invariants [eqs. (4) and (12)].

$$
\begin{aligned}
W= & \frac{N_{s}^{\sim}}{2}\left(\frac{A_{\alpha \eta}}{X_{\alpha \lambda} Y_{\eta \lambda}} Z_{\eta \lambda}+\ln \left(Y_{\eta \lambda}\right)+\ln \left(X_{\alpha \lambda}\right)\right) \\
& +\frac{N_{c}^{\sim}}{2}\left(\frac{\left(1-\alpha^{2}\right)}{X_{\alpha \lambda}} I_{1}^{e}+\ln \left(X_{\alpha \lambda}\right)\right) \\
& Y_{\eta \lambda}=1+\eta I_{1}^{e}+\eta^{2} I_{2}^{e}+\eta^{3} ; X_{\alpha \lambda}=1-\alpha^{2} I_{1}^{e} ; \\
& Z_{\eta \lambda}=I_{1}^{e}+2 \eta I_{2}^{e}+3 \eta^{2} ; A_{\alpha \eta}=(1+\eta)\left(1-\alpha^{2}\right)
\end{aligned}
$$

For convenience, let us introduce, $n_{c}$, the ratio $\mathrm{N}_{\mathrm{c}} /$ $\mathrm{N}_{\mathrm{s}}$, that represents the level of crosslinkage of the network. Obviously, this model only allows reproducing hyperelastic behaviors but is rather powerful despite of a few numbers of parameters.

Time dependent effects can be introduced through the above-proposed route (alteration of parameters). Let us imagine that any elementary process that modifies microstructure results in a change in one or all of the four parameters, in example:

- Disentanglement could result in an increase in $\eta$;

- Crystallization could result in an increase in $n_{c}$ and/or an increase in $\alpha^{2}$, which means that crosslinks are more numerous and chains are less extensible, respectively. One can also imagine that $N_{s}^{\sim}$ decreases in parallel to those latter effects.

Hence, according to eq. (10):

$$
\begin{array}{r}
\frac{\partial \varepsilon^{a}}{\partial t}=\frac{\beta^{\prime}}{\sigma}\left(\frac{\partial W}{\partial \eta} \frac{\partial \eta}{\partial t}+\frac{\partial W}{\partial n_{c}} \frac{\partial n_{c}}{\partial t}+\frac{\partial W}{\partial N_{s}^{\sim}} \frac{\partial N_{s}^{\sim}}{\partial t}+\frac{\partial W}{\partial \alpha^{2}} \frac{\partial \alpha^{2}}{\partial t}\right) \\
\text { with } \quad \beta^{\prime} \geq 1
\end{array}
$$

In consequence, last point to address is the description of the kinetics for those alterations. At this stage, let us first have a phenomenological 
approach. Obviously, rate of microstructure changes must be related to the energy that is available in the material. If one focus on disentanglements and strain induced crystallization, one can assume that the more stressed the slip-links the faster the processes. In consequence, this means that that kinetics should probably depend on the elementary energy of the slip-link due to extension, $f_{s}$ [eq. (14)].

$$
\begin{aligned}
\mathrm{f}_{\mathrm{s}}= & \left(\frac{\mathrm{A}_{\alpha \eta}}{\mathrm{X}_{\alpha \lambda} \mathrm{Y}_{\eta \lambda}} \mathrm{Z}_{\eta \lambda}+\ln \left(\mathrm{Y}_{\eta \lambda}\right)+\ln \left(\mathrm{X}_{\alpha \lambda}\right)\right) \\
& -3\left[\frac{\left(1-\alpha^{2}\right)}{\left(1-3 \alpha^{2}\right)}+\ln (1+\eta)\right]-\ln \left(1-3 \alpha^{2}\right)
\end{aligned}
$$

Then, if one assumes that:

- Disentanglement could result in: $\frac{\partial \eta}{\partial t}=\Im_{1}\left(f_{s}\right)>0$

- Crystallization could result in: $\frac{\partial n_{c}}{\partial t}=\Im_{2}\left(f_{s}\right)>0$ and/or: $\quad \frac{\partial \alpha^{2}}{\partial t}=\Im_{3}\left(f_{s}\right)>0$ and eventually: $\frac{\partial N_{s}^{\sim}}{\partial t}=\Im_{4}\left(f_{s}\right)>0$.

- Behavior is ruled by the functions $\Im_{j}(j=1, . ., 4)$ ) that are combined with eqs. (14) and (12) [or eq. (13)] and, finally eq. (1). Some examples are illustrated below.

\section{NUMERICAL APPLICATION}

An explicit scheme is adopted to calculate the evolution of the stress as a function of the strain-rate. At any time, $t$, of a uniaxial loading of an incompressi- ble polymer, principal global extension ratios are $\lambda,+\frac{1}{\sqrt{\lambda}}$ and $-\frac{1}{\sqrt{\lambda}}$. Due to the coexistence of elastic and inelastic components, $\lambda^{\mathrm{e}}$ and $\lambda^{\mathrm{a}}$, respectively: $\lambda(\mathrm{t})=\lambda^{\mathrm{e}}(\mathrm{t}) \lambda^{\mathrm{a}}(\mathrm{t})$. Uniaxial stress is given by eqs. (2) and (11) as a function of $\lambda^{\mathrm{e}}(\mathrm{t})$. In the same manner, $f_{s}(t)$ is ruled by eq. (14) and depends on the same parameter $\lambda^{\mathrm{e}}(\mathrm{t})$. For both those two functions, one has to consider the instantaneous values for parameters $N_{s}^{\sim}(t), n_{c}(t), \alpha^{2}(t)$ and $\eta(t)$. In addition, at any time, $t$ :

$$
\begin{aligned}
& \frac{\partial \eta}{\partial t}(t)=\Im_{1}\left(f_{s}\left(\lambda^{e}(t)\right)\right) ; \frac{\partial n_{c}}{\partial t}(t) \\
&=\Im_{2}\left(f_{s}\left(\lambda^{e}(t)\right)\right) ; \frac{\partial \alpha^{2}}{\partial t}(t)=\Im_{3}\left(f_{s}\left(\lambda^{e}(t)\right)\right)
\end{aligned}
$$

that induces the inelastic strain-rate $\frac{\partial \varepsilon^{a}}{\partial t}(t)$, given by:

$$
\begin{array}{r}
\frac{\partial \varepsilon^{a}}{\partial t}(t)=\frac{\beta^{\prime}}{\sigma\left(\lambda^{e}(t)\right)}\left(\frac{\partial W(t)}{\partial \eta} \frac{\partial \eta}{\partial t}(t)+\frac{\partial W(t)}{\partial n_{c}} \frac{\partial n_{c}}{\partial t}(t)\right. \\
\left.+\frac{\partial W(t)}{\partial N_{s}^{\sim}} \frac{\partial N_{s}^{\sim}}{\partial t}(t)+\frac{\partial W(t)}{\partial \alpha^{2}} \frac{\partial \alpha^{2}}{\partial t}(t)\right)
\end{array}
$$

For simplicity, let us consider that the total density of slip-links and crosslinks remains constant. This assumption, which is not compulsory, implies that $\frac{\partial N_{s}^{\sim}}{\partial t}(t)=-\frac{N_{s}^{\sim}(t)}{\left(1+n_{c}(t)\right)} \frac{\partial n_{c}}{\partial t}(t)$.

During time step, $d t$, at the strain-rate $\frac{\partial \varepsilon}{\partial t}(t)$, Henky's strain increases by $d \varepsilon=\frac{\partial \varepsilon}{\partial t}(t) d t$. In consequence,

$$
\left\{\begin{array}{l}
\lambda(t+d t)=\lambda(t) \exp \left(\frac{\partial \varepsilon}{\partial t}(t) d t\right) ; \quad \lambda^{a}(t+d t)=\lambda^{a}(t) \exp \left(\frac{\partial \varepsilon^{a}}{\partial t}(t) d t\right) ; \quad \lambda^{e}(t+d t)=\frac{\lambda(t+d t)}{\lambda^{a}(t+d t)} \\
\eta(t+d t)=\eta(t)+\frac{\partial \eta}{\partial t}(t) d t ; n_{c}(t+d t)=n_{c}(t)+\frac{\partial n_{c}}{\partial t}(t) d t ; \alpha^{2}(t+d t)=\alpha^{2}(t)+\frac{\partial \alpha^{2}}{\partial t}(t) d t \\
N_{s}^{\sim}(t+d t)=\frac{N_{s}^{\sim}(0)+N_{c}^{\sim}(0)}{1+n_{c}(t+d t)} \\
W(t+d t)=W\left(\lambda^{e}(t+d t), \eta(t+d t), n_{c}(t+d t), \alpha^{2}(t+d t), N_{s}^{\sim}(t+d t)\right) \\
\sigma(t+d t)=\lambda^{e}(t+d t) \frac{\partial W}{\partial \lambda^{e}}(t+d t)
\end{array}\right.
$$

Initial time increment is assumed to be totally elastic, which implies $\lambda^{e}(t=d t)=\lambda(t=d t)$.

Figure 1 depicts results obtained in two fictitious 0.01 and $0.001 \mathrm{~s}^{-1}$ uploading-unloading cycles and allows illustrating and comparing effects of alteration of parameters one by one. In those cases, $\Im_{j}$ functions of eqs. (15) are "simple" [eq. (18)] and are chosen in an arbitrary manner. At this stage, we only intend to give an example only accounting for the fact that alteration kinetics is faster when energy increases, using as less numerous parameters as possible (one to two).

$$
\begin{aligned}
& \Im_{j}=\xi_{j}\left(\exp \left(\mathrm{f}_{\mathrm{s}}-\Psi_{\mathrm{j}}\right)-1\right) \quad \text { if } \quad \mathrm{f}_{\mathrm{s}}>\Psi_{\mathrm{j}} \\
& \Im_{\mathrm{j}}=0 \quad \text { if } \quad \mathrm{f}_{\mathrm{s}} \leq \Psi_{\mathrm{j}}
\end{aligned}
$$

Despite of that simplicity, the model potentially allows reproducing most of the shapes observed for tensile tests in polymers, which are often associated to plasticity, viscoelasticity, softening, hardening, and damaging. Figure 1(b), for its part, demonstrates that, introducing the kinetics of microstructure modification makes it possible to predict some sensitivity to strain-rate without a priori dependence of parameters. Finally, Figure 2 depicts our ability to 

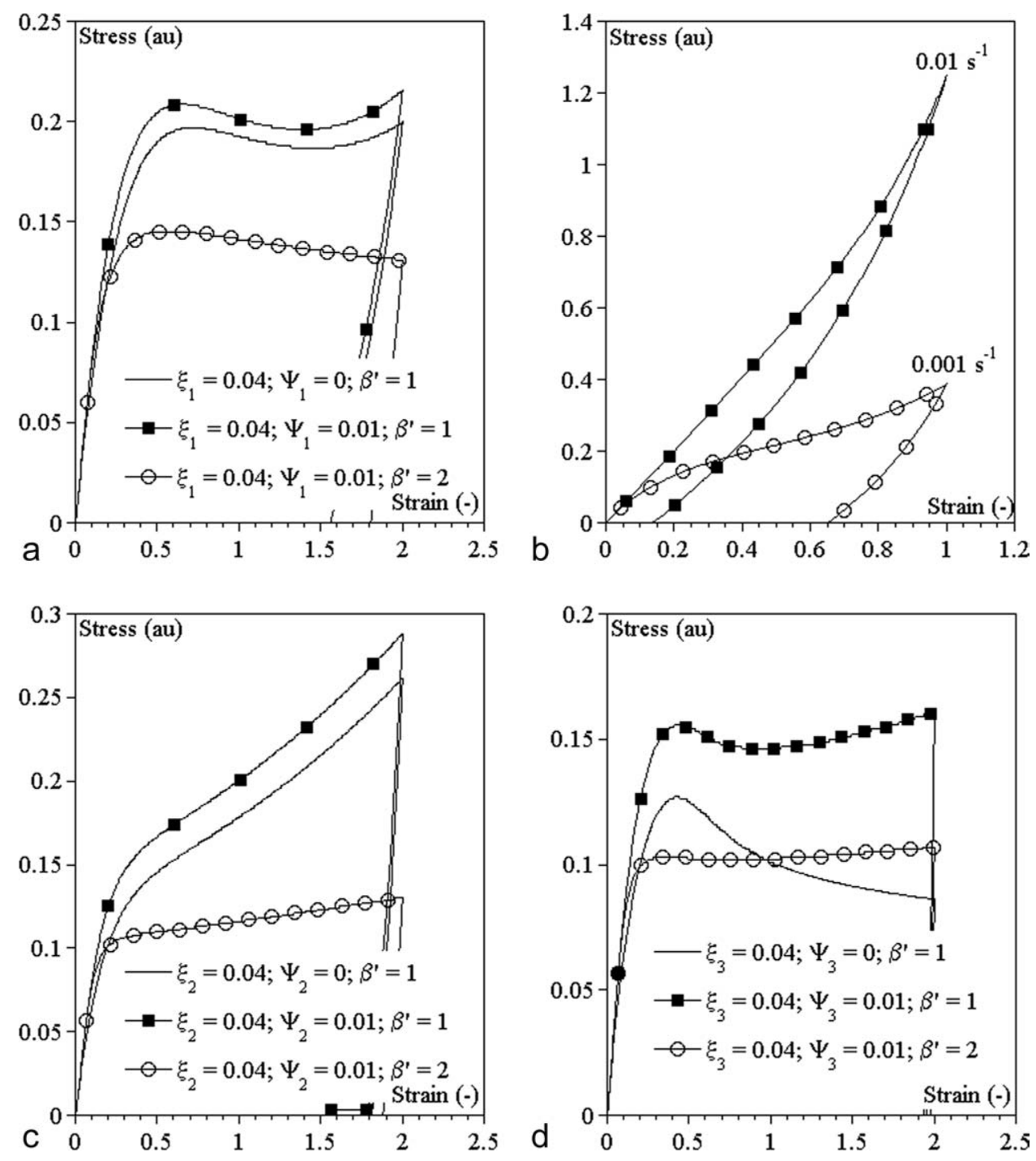

Figure 1 Efficiency of new approach to model uploading-unloading cycles for a constant strain-rate; (a) Effects of an increase in $\eta$ at strain-rate of $\pm 0.01 \mathrm{~s}^{-1}$ for $n_{c}=0.1, \alpha^{2}=0.001$, and $\eta(0)=0.2343$; (b) Effect of strain-rate ( \pm 0.0001 and $\pm 0.01 \mathrm{~s}^{-1}$ ) for $n_{c}=1, \alpha^{2}=0.001, \eta(0)=0.2343, \xi_{1}=0.01, \Psi_{1}=0$, and $\beta^{\prime}=1$; (c) Effects of an increase $n_{c}$ at strain-rate of $\pm 0.01 \mathrm{~s}^{-1}$ for $n_{c}(0)=0.1, \alpha^{2}=0.001$, and $\eta=0.2343$; (d) Effects of increase in $\alpha^{2}$ at strain-rate of $\pm 0.01 \mathrm{~s}^{-1}$ for $n_{c}=0.1$, $\alpha^{2}(0)=0.001$, and $\eta=0.2343$.

reproduce uploading-unloading-uploading loops compared with initial EV's model.

One has to emphasize that only a few numbers of parameters (5-6 per conditions) are necessary to obtain such a good efficiency in introducing time effects in constitutive model, which makes this model unique. The question is than, is the model relevant for polymers? This is further validated using experimental results obtains on a PMMA.

\section{EXPERIMENTAL VALIDATION}

\section{Material and protocol}

The material of this study was a poly(methyl methacrylate) (PMMA) ALTUGLAS V $825 \mathrm{~T}$ provided by Arkema. Average molar masses, $M_{n}$ and $M_{w}$, were
40,434 and 93,000 g/mol, respectively. For its part, polydispersity index $Q$, was 2.3. Material was processed as $4 \mathrm{~mm}$-thick injection molded plaques of dimensions $10 \mathrm{~cm}$ by $10 \mathrm{~cm}$. Gate was a throughthe-width lateral gate and processing conditions were chosen according to the state of the art. Resulting mechanical anisotropy in the plane of the plaques was found to be negligible.

DMTA analyses were performed in tension on 5 $\mathrm{mm}$ by $1 \mathrm{~mm}$ bars, to additionally characterize the linear viscoelastic domain of the material (maximum strain of $2 \times 10^{-3}$ ). The $\alpha$-transition temperature (chosen at the maximum value of the tangent of the loss angle) ranged from 128 to $146^{\circ} \mathrm{C}$, for loading frequencies ranging from 0.3 to $31 \mathrm{~Hz}\left(132^{\circ} \mathrm{C}\right.$ for a 1 $\mathrm{Hz}$-frequency). Despite of some discrepancy for higher frequencies, the time-temperature 


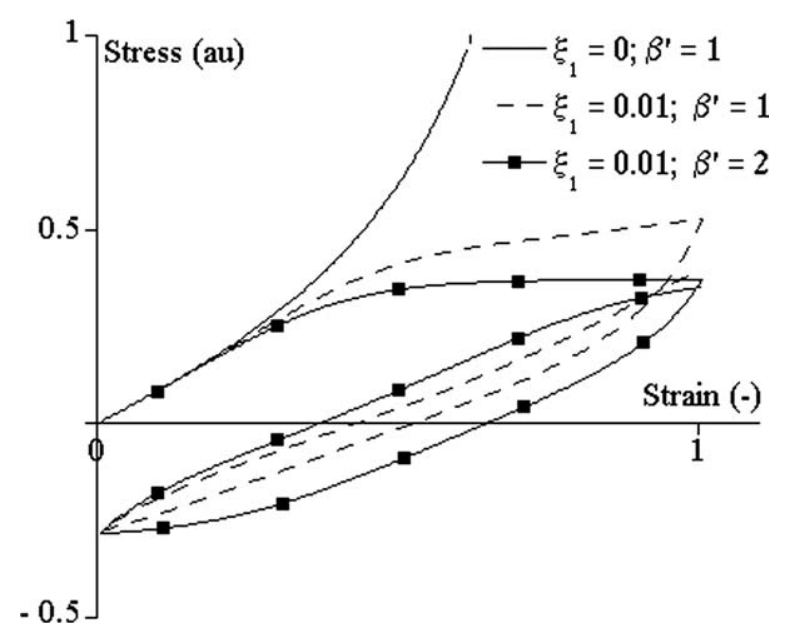

Figure 2 Comparison of new model to initial EV's model referred to as $\left(\zeta_{1}=0, \beta^{\prime}=1\right)$ in the case where $n_{c}=0.1$, $\alpha^{2}=0.1, \eta(0)=0.2343$, and $\frac{\partial \eta}{\partial t}=\zeta_{1} f_{s}^{2}$.

superposition principle could be applied. A master curve was built up (from 90 to $180^{\circ} \mathrm{C}$; Fig. 3) that involved one unique horizontal shift factors, $a_{T / T_{0}}$, identical for both the storage and the loss modules. To summarize, for the reference temperature of $130^{\circ} \mathrm{C}, a_{T / T_{0}}$ obeyed eq. (19).

$$
\lg _{10}\left(a_{T / T_{0}}\right)=\frac{-7.1 \quad(T(K)-403)}{51.8+T(K)-403}
$$

In a second step, tensile behavior was intensively explored at temperature ranging from 100 to $160^{\circ} \mathrm{C}$. Entire experimental database consists of uploading (up to different levels of strain)-unloading or uploading (up to different levels of strain)-relaxation experiments, each performed at strain-rates ranging from $10^{-4} \mathrm{~s}^{-1}$ to $9 \mathrm{~s}^{-1}$. To achieve that point samples

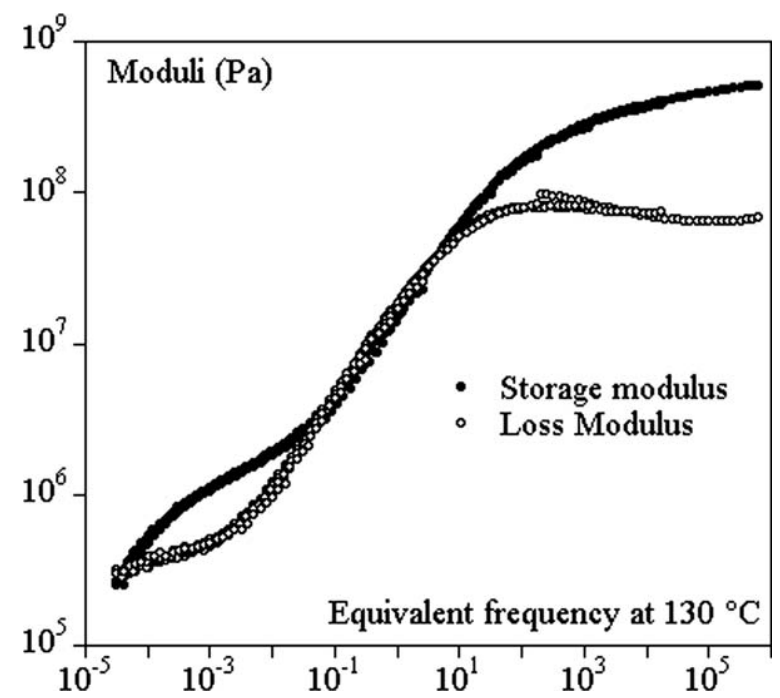

Figure 3 Master curve for the PMMA used in the study. Reference temperature is $130^{\circ} \mathrm{C} ; \mathrm{C}_{1}=7.1 ; \mathrm{C}_{2}=51.8$.

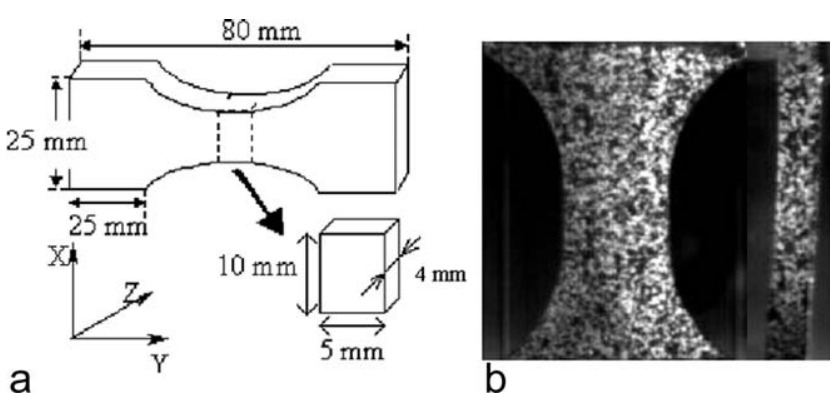

Figure 4 (a) Schematics of the tensile samples; (b) Random patterns on the front (left) face and on the lateral face (right) as observed using a mirror.

[Fig. 4(a)] having a small rectilinear process zone, which allowed concentrating truly uniaxial loading conditions, were machined perpendicular to the flow direction. Local strains were measured using, either full fields strain measurements [based on random patterns painted on surface, Fig. 4(b)], or a four dots video extensometer method. Using mirrors, it was possible to analyze strain fields on both the front face and the lateral face of the samples [Fig. $4(b)]$, resulting in a 3D measurements. As a conclusion, let us emphasizes that experimental database is as complete and as rigorous as possible.

\section{Experimental}

The entire database is schematically described in Figure 5. However, in this article we refer to experiments that were conducted between 120 and $150^{\circ} \mathrm{C}$ and from $10^{-4}$ to $0.01 \mathrm{~s}^{-1}$ (Trials $1-10$ ), which are conditions corresponding to the rubbery plateau for the chosen PMMA. We also restrain our present analysis to strain-rates lower than $0.01 \mathrm{~s}^{-1}$, to avoid

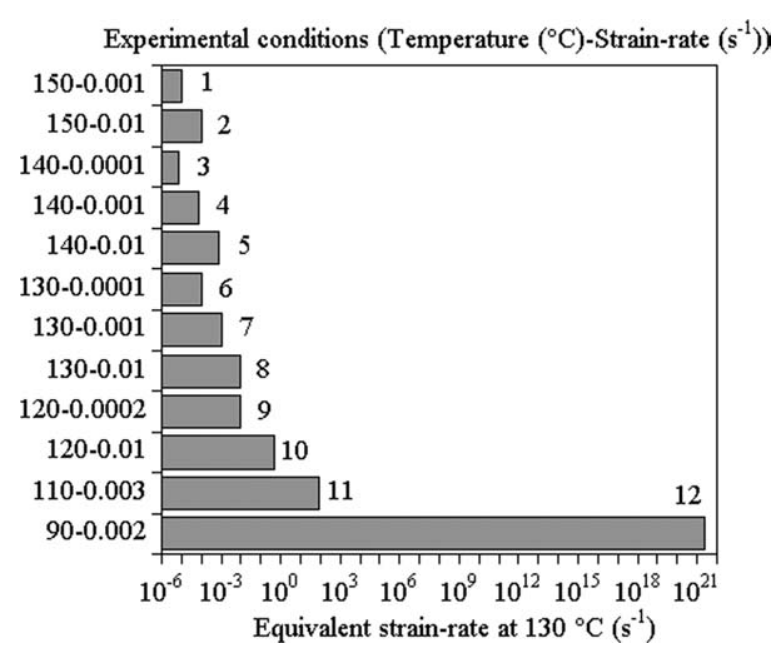

Figure 5 Schematics of the experimental database and associated equivalent strain-rate at $130^{\circ} \mathrm{C}$. Numbers refers to trials. 
any disturbance related to thermomechanical coupling. Nevertheless, experimental data that is used here corresponds to equivalent strain-rates ranging from 0.5 down to $10^{-6} \mathrm{~s}^{-1}$ at $130^{\circ} \mathrm{C}$, which is over five decades of strain-rates.

In that range, 3D extensometers allowed concluding that the plaques were totally isotropic, which means that strain fields on the front face were equivalent to that on the lateral face of the samples. Additionally, the volume strain that resulted from the three components of the strain tensor remained close to zero in any case. Consequently, this PMMA is an isotropic and incompressible material and can be modeled using our model.

In the following sections, typical behavior of PMMA is depicted thanks to the so-called true uniaxial stress (i.e., referring to the actual section at any time) versus longitudinal Henky's strain, $\bar{\varepsilon}$, or extension ratio, $\lambda=\exp (\bar{\varepsilon})$. Numbers in the legends refer to the number of trial as defined in Figure 5.

As expected, behavior of PMMA is significantly dependent upon strain-rate and temperature in the range that was considered in this study. In a less classical manner, it was observed that equivalent strain-rate at reference temperature was a relevant parameter to refer to experimental conditions. As a proof, compare Trials 1 and 3 in Figures 6 (hollow symbols) and 5; Trials 4 and 6 in Figures 6 (hollow symbols) and 5; Trials 8 and 9 in Figures 7 (hollow symbols) and 5.

\section{Validation of the model}

The model described in the above sections could reproduce those experimental data. To achieve that point, parameters of the model were identified as a

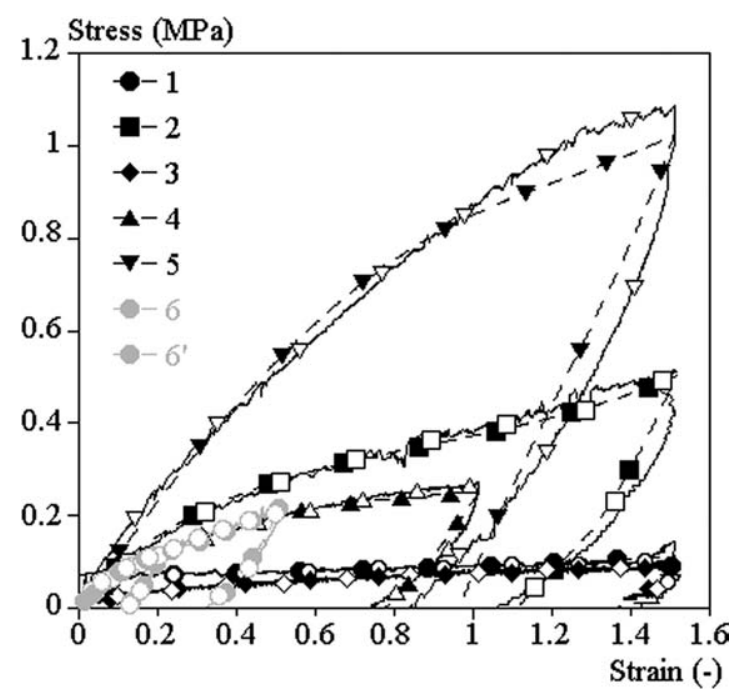

Figure 6 Comparison between experiments (opens symbols) and models (solid symbols). Identification was done curve by curve (Table I) and numbers refer to Figure 5.

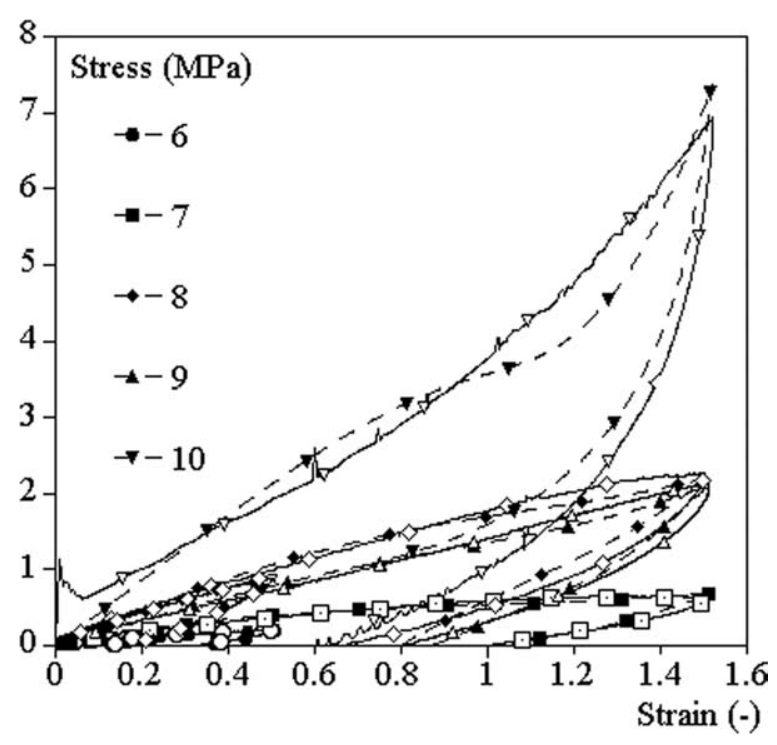

Figure 7 Comparison between experiments (opens symbols) and models (solid symbols). Identification was done curve by curve (Table I) and numbers refer to Figure 5.

whole set using inverse analysis. Cost function was defined in a classical "mean square" method based on experimental and calculated stresses. Minimization relied on the simplex algorithm of the "fminsearch" MATLAB® function.

It was only necessary to model an increase in $\eta$, that is, disentanglement, to reproduce experimental observations. A purely phenomenological approach was chosen [eq. (20)].

$$
\begin{aligned}
\frac{\partial \eta}{\partial t}=\xi( & \left.\exp \left(f_{s}^{p}\right)-1\right)-\mu g_{s}\left(1-\exp \left(-f_{s}\right)\right) \\
g_{s} & =\left(\frac{A_{\alpha \eta}}{X_{\alpha \lambda} Y_{\eta \lambda}} Z_{\eta \lambda}+\ln \left(Y_{\eta \lambda}\right)+\ln \left(X_{\alpha \lambda}\right)\right) \\
& -\left(\frac{A_{\alpha \eta_{0}}}{X_{\alpha \lambda \lambda} Y_{\eta_{0} \lambda}} Z_{\eta_{0} \lambda}+\ln \left(Y_{\eta_{0} \lambda}\right)+\ln \left(X_{\alpha \lambda}\right)\right)
\end{aligned}
$$

$\xi, P$, and $\mu$ are parameters. For its part, $g_{s}$ is the excess of elementary energy due to the increase of $\eta$ at the given extension. The associate kinetics term accounts for the fact that global evolution of slipperiness factor must result from the balance between disentanglement, induced by straining, and reentanglement, induced by the fact that $\eta$-value is not the equilibrium value.

First attempt consisted in the identification of parameters curve-by-curve (Figs. 6 and 7). In any case, it was possible to reproduce experimental curve with a good agreement using only six parameters. Looking carefully to $y$-scales one observes that consistency of the polymer drastically varied in the considered experimental range, which is, polymers exhibited behavior ranging from a close to glassy state material (Trial 10) to an almost fluid material (Trial 1). 
TABLE I

Parameters Identified Experimental Condition by Experimental Conditions

\begin{tabular}{|c|c|c|c|c|c|c|c|c|}
\hline Trial (Fig. 5) & $\begin{array}{l}N_{s}^{\sim} \\
(\mathrm{MPa})\end{array}$ & $\begin{array}{c}N_{c}^{\sim} \\
(\mathrm{MPa})\end{array}$ & $\alpha^{2}$ & $\xi\left(\times 10^{-3}\right)$ & $P$ & $\mu$ & $N_{s}\left(\times 10^{25} \mathrm{~m}^{-3}\right)$ & $N_{c}\left(\times 10^{25} \mathrm{~m}^{-3}\right)$ \\
\hline 1 & 0.23 & 0.23 & $6 \times 10^{-5}$ & 1.19 & 1.25 & 0.07 & 4 & 6.0 \\
\hline 2 & 0.53 & 0.16 & $10^{-4}$ & 0.23 & 0.69 & 0.0002 & 9.1 & 4.1 \\
\hline 3 & 0.19 & 0.04 & $4 \times 10^{-5}$ & 0.01 & 0.63 & 0.006 & 3.3 & 1.1 \\
\hline 4 & 0.39 & 0.08 & $9 \times 10^{-5}$ & 0.1 & 0.74 & $10^{-8}$ & 6.9 & 2.1 \\
\hline 5 & 0.98 & 0.21 & $2 \times 10^{-4}$ & 3 & 1 & 2 & 17.2 & 5.7 \\
\hline 6 & 0.13 & 0.26 & $10^{-3}$ & 4 & 1.1 & 16 & 2.3 & 6.9 \\
\hline 7 & 0.73 & 0.14 & $3 \times 10^{-4}$ & 1 & 0.65 & $5 \times 10^{-8}$ & 13 & 3.9 \\
\hline 8 & 0.62 & 0.39 & $7 \times 10^{-4}$ & 40 & 1.2 & 2 & 11 & 10.3 \\
\hline 9 & 1.46 & 0.39 & $7 \times 10^{-4}$ & 9 & 0.5 & 0.0004 & 27 & 10.4 \\
\hline 10 & 1.72 & $\sim 0$ & $2 \times 10^{-2}$ & 0.01 & 1 & 2 & 32 & $\sim 0$ \\
\hline
\end{tabular}

Despite of this huge evolution in behavior and despite of a certain scattering, parameters (Table I) vary in a coherent manner with equivalent strainrate at reference temperature. Consequently, second step was to introduce dependences upon temperature and strain-rate in a combine manner using WLF's approach. In fact, experimental observations proved the relevance of such a route [eq. (21)]. According to Table I, it was chosen to assume that $N_{s} \backslash \operatorname{sim}, N_{c} \backslash \operatorname{sim}, \xi$, and $\beta^{\prime}$ depend upon average equivalent strain-rate, according to the same type of equation [eq. (21)]. Two pseudo-relaxation times, $\tau_{1}$ and $\tau_{2}$, were introduced.

$$
\begin{aligned}
\left\{\begin{array}{c}
N_{s}^{\sim} \\
N_{c}^{\sim} \\
\xi \\
\beta^{\prime}
\end{array}\right\}= & \left\{\begin{array}{c}
N_{s 0}^{\sim} \\
N_{c 0}^{\sim} \\
\xi_{0} \\
1
\end{array}\right\}+2\left\{\begin{array}{c}
N_{s 1}^{\sim} \\
N_{c 1}^{\sim} \\
\xi_{1} \\
\beta_{1}^{\prime}
\end{array}\right\} \frac{1}{1+\left(\tau_{1} a_{T / T_{0}} \dot{\varepsilon}\right)^{-2 m_{1}}} \\
& +2\left\{\begin{array}{c}
N_{s 2}^{\sim} \\
N_{c 2}^{\sim} \\
\xi_{2} \\
\beta_{2}^{\prime}
\end{array}\right\} \frac{1}{1+\left(\tau_{2} a_{T / T_{0}} \dot{\varepsilon}\right)^{-2 m_{2}}}
\end{aligned}
$$

In parallel to that, as the extensibility of the chains should be partly ruled by the level of cross linkage of the network, $\alpha^{2}$ was assumed to be proportional to $N_{c}^{\sim}$. Finally, $P$ (power of the disentanglement factor), was found to linearly depend upon strain-rate ( $\left.p=p_{0}+p_{1} a_{T / T_{0}} \dot{\varepsilon}\right)$, whereas $\mu$ (reentanglement factor) remained close to 0 .

Parameters are summarized in Table II and Figure 8. They account for the fact that the higher the strain-rate or the lower the temperature, the more rigid the network and the higher the dissipation.

According to this first attempt mechanical behavior of PMMA above, but close to $T_{g}$, can be reproduced modeling this polymer as a entangled network of entanglement mobility depending upon straining, temperature, and strain-rate. Density of entanglements, $N_{s}$, drastically increases close to $\alpha$ transition. Density of apparently permanent nodes,
$N_{c}$, also increases but to a smaller extent. However, this latter remains significantly lower to $N_{s}$. These points are acceptable for an amorphous polymer.

In parallel, disentanglement parameter, $\xi$, increases. However, it is difficult to relate this parameter to a simple phenomenon. It rules the part of mechanical power that is converted into inelastic phenomenon at a given level of elastic energy: that is, the higher the strain-rate or the lower the temperature, the more power is dissipated into inelasticity at any time. This is also consistent with experimental observation close to $T_{g}$ on the rubbery plateau.

Finally, the balance between reversible mechanical power (elastic, related to $N_{s}$ and $N_{c}$ ) and dissipation mechanical power (related to $\xi$ and $\beta^{\prime}$ ) is controlled by an increase in relative importance of dissipative phenomenon at low temperature (resp. high strainrate) and a decrease in relative importance of elastic phenomenon at high temperature (resp. low strainrate).

TABLE II

Parameters of the Model Accounting for Dependence

\begin{tabular}{|c|c|c|}
\hline Parameter & Value & Unit \\
\hline$N_{s 0}^{\sim}$ & 0.0048 & $\mathrm{MPa}$ \\
\hline$N_{c 0}^{\sim}$ & 0.00066 & $\mathrm{MPa}$ \\
\hline$\xi_{0}$ & $7.55 \times 10^{-9}$ & - \\
\hline$N_{s 1}^{\sim}$ & 0,53 & $\mathrm{MPa}$ \\
\hline$N_{c 1}^{\sim}$ & $8.7 \times 10^{-8}$ & $\mathrm{MPa}$ \\
\hline$\xi_{1}$ & 0,026 & - \\
\hline$\beta_{1}^{\prime}$ & $9.5 \times 10^{-5}$ & - \\
\hline$N_{s 2}^{\sim}$ & 0.33 & $\mathrm{MPa}$ \\
\hline$N_{c 2}^{\sim}$ & 252 & $\mathrm{MPa}$ \\
\hline$\xi_{2}$ & $3.6 \times 10^{-5}$ & - \\
\hline$\beta_{2}^{\prime}$ & 3.21 & - \\
\hline$p_{0}$ & $7.55 \times 10^{-9}$ & - \\
\hline$p_{1}$ & 45.6 & $\mathrm{~s}$ \\
\hline$\tau_{1}$ & 8.6 & $\mathrm{~s}$ \\
\hline$m_{1}$ & 0.488 & - \\
\hline$\tau_{2}$ & 4004 & $\mathrm{~s}$ \\
\hline$m_{2}$ & 0.224 & - \\
\hline$\frac{\alpha^{2}}{N_{c}^{\sim}}$ & $9 \times 10^{-6}$ & - \\
\hline
\end{tabular}
Upon Temperature and Strain-Rate 


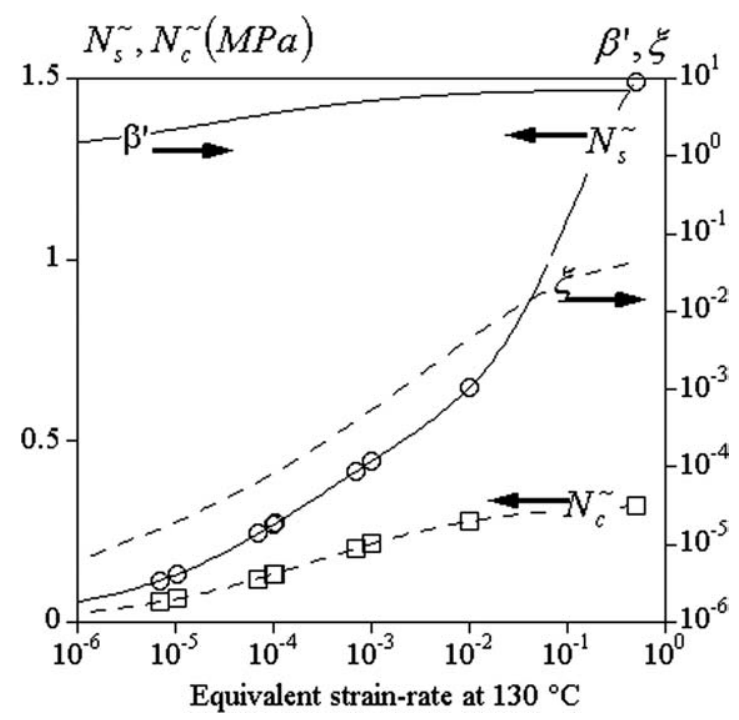

Figure 8 Parameters of the models (Table II) versus equivalent strain-rate at $130^{\circ} \mathrm{C}$. Symbols represent values corresponding to experimental database.

Anyway, this partly phenomenological approach, combined with our novel model, allowed reproducing the behavior of PMMA from 0.01 to $0.0001 \mathrm{~s}^{-1}$, and from 120 to $150^{\circ} \mathrm{C}$ (Figs. 9-13) and potentially from $10^{-6}$ to $0.5 \mathrm{~s}^{-1}$ in terms of equivalent strainrate at reference temperature, using one unique set of parameters.

To finish, it was also possible, using the same set, to reproduce both uploading-unloading and uploading-relaxation experiments (Fig. 14). In conclusion, the new model made it possible to reproduce most of the viscoelastic characteristics of a rubbery PMMA and their dependence upon temperature and strainrate only defining a few number of parameters.

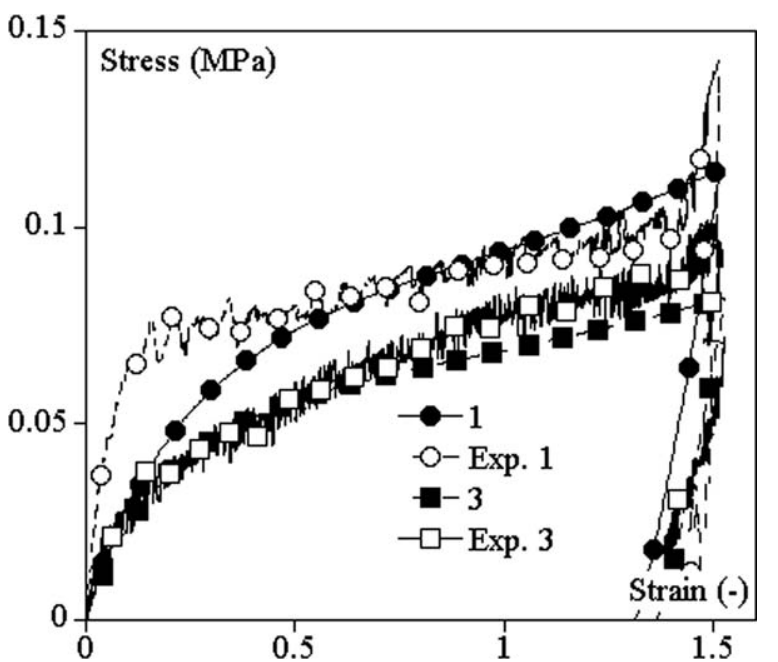

Figure 9 Comparison between experiment and global model using data of Table II for Cases 1 and 3 of Figure 5.

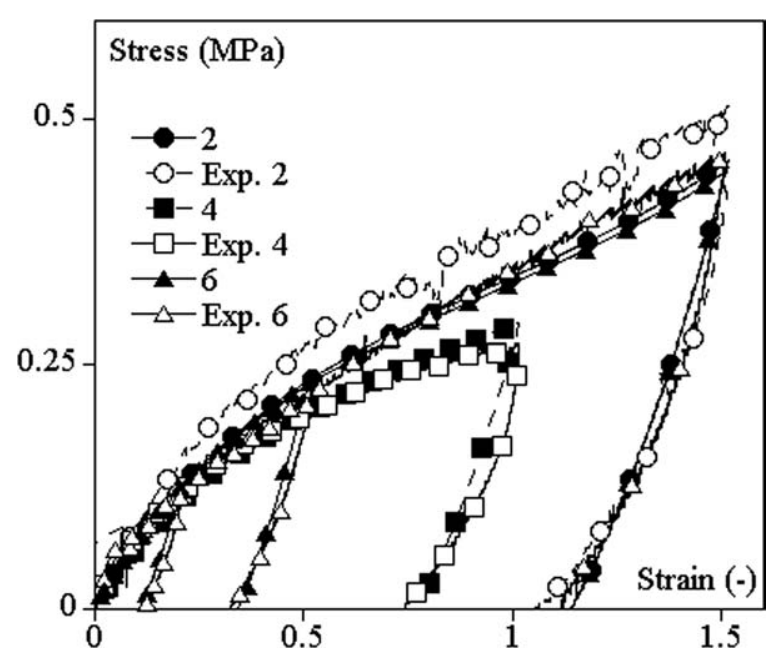

Figure 10 Comparison between experiment and global model using data of Table II for Cases 2, 4, and 6 of Figure 5.

\section{DISCUSSION}

Despite of the good agreement observed above, one could argue that permanent nodes are not assumed to exist in PMMA above $T_{g}$. So should not $N_{c}$ be equal to zero? Unfortunately, this would not be coherent with initial assumption in EV's model. So is the model physically relevant in the case of amorphous polymers?

In fact, those network models are not deterministic but statistical approaches. Indeed, one has to consider only that EV'S model is a virtual simplified medium whose behavior, for example, Helmozt's free energy is statistically equivalent to that of the actual material. Consequently, chains are not molecules per se, and permanent nodes must not only be considered as individual chemical crosslinks.

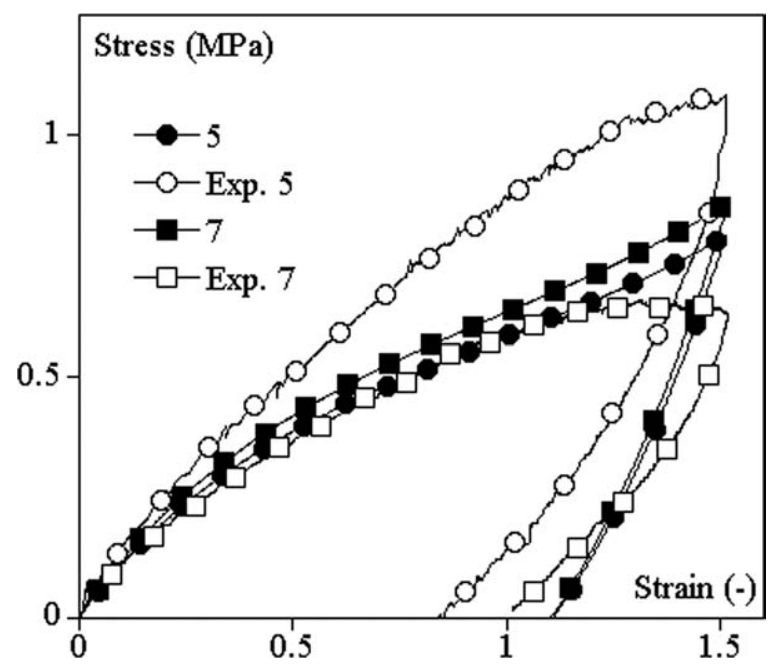

Figure 11 Comparison between experiment and global model using data of Table II for Cases 5 and 7 of Figure 5. 


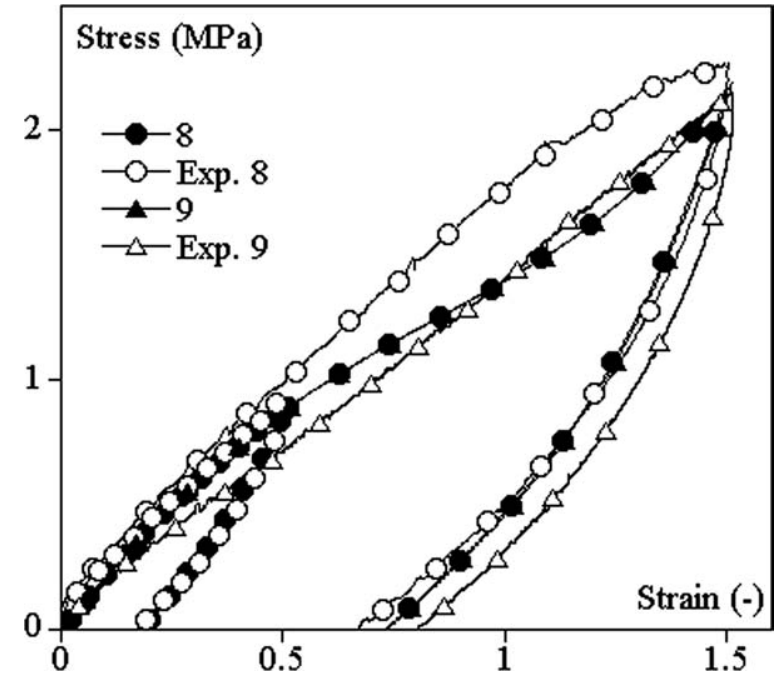

Figure 12 Comparison between experiment and global model using data of Table II for Cases 8 and 9 of Figure 5.

More precisely, there is no reason for all the physical entanglements to exhibit the same level of mobility. Some should exist with highly cooperative van der Waals-type interactions or/and important topological constraints, making the molecules apparently connected to each other, at least in the considered loading conditions. Therefore, exact assumption is that this distribution in entanglements mobility can be reduced in a bimodal distribution of statistical nodes: some can be grouped in a family having a $\eta=0.2343$ average value, other can be grouped in a family having a $\eta=0$ average value. In consequence, this model can be relevant even for amorphous polymers.

Validity domain should be above $T_{g}$ as initial EV's model. More rigorously, the phenomenological use of time temperature superposition principle makes it valid above $T_{\alpha}$ that is up to high strain rate for higher temperature and/or down to low strain rate

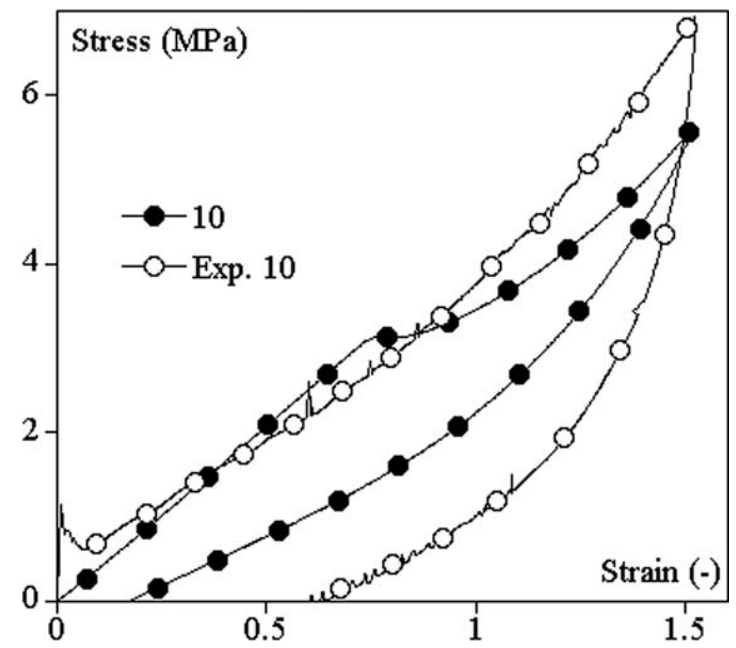

Figure 13 Comparison between experiment and global model using data of Table II for Case 10 of Figure 5.

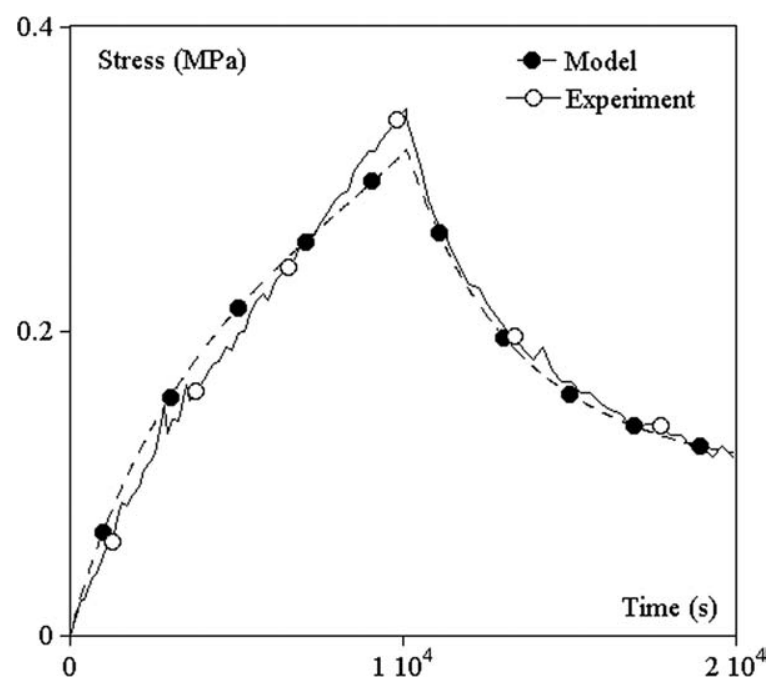

Figure 14 Comparison between experiment (open symbols) and model (solid symbols) in the case of uploadingrelaxation at $130^{\circ} \mathrm{C}$ and $0.0001 \mathrm{~s}^{-1}$.

for temperature close to $T_{g}$. In fact, its validity must be expressed in terms of equivalent strain rate at reference temperature. In case of PMMA, it was checked from 0 (relaxation) to $1 \mathrm{~s}^{-1}$.

\section{CONCLUSION}

An innovative visco-hyperelastic model has been developed and validated for modeling of the behavior of polymers in their rubbery state.

In this approach, mechanical behavior is not decomposed into different components through an "a priori" combination as usually. On the contrary, inelastic phenomenon derives from the alteration of internal variables assumed to be related to the microstructure, which induces changes in well-chosen constitutive parameters.

Inelastic strain-rate is such as energy of the material remains constant, that is, energy necessary for changes in the microstructure is balanced by the release in elastic energy and other dissipative effects. This concept allowed introducing time effects in the rubber elasticity theory, resulting in visco-hyperelastic, visco-plastic model consistent with continuum thermodynamics and obeying second principle by construction. The approach has been entirely developed based on the EV's theory but could be extended to any other model.

In parallel, simple modeling of disentanglement or crystallization allowed reproducing most of the viscoelastic phenomenon observed in polymers: uploading-unloading loops, relaxation and apparent dependence upon strain-rate. It must be emphasized that this was achieved using one unique formulation, which is, the model is general enough to reproduce all these effects using one set of parameters. 
It is suggested that accounting for temperature and strain-rate effects by an "a priori" uses of time temperature superposition principle could be a pleasant route for developing simpler model. Feasibility of such opportunity is clearly demonstrated in this article on the base of a rich experimental database obtained for a PMMA. Authors have performed equivalent work on HIPS, PU, PET, and even PA 66 that will be further reported. As a first approach phenomenological kinetics law were proposed. Further works will be devoted to develop more physically based modeling.

However, at this stage this model represents a highly attractive way of modeling time dependent behavior of polymers over a wide range of temperature and strain-rate up to of large strains. Despite of the fact that we only presented a 1D limit of the model, this later is a fully 3D model that could be used in finite element simulations.

\section{REFERENCES}

1. Chaboche, J.-L. Int J Solids Struct 1997, 34, 2239.

2. Germain, P.; Nguyen, Q.; Suquet, P. J Appl Mech 1983, 50, 1010 .

3. Bergström, J. S.; Rimnac, C. M.; Kurtz, S. M. Biomaterials 2003, 24, 1365.

4. Bergström, J. S.; Rimnac, C. M.; Kurtz, S. M. Biomaterials 2004, 25, 2171.

5. Bergström, J. S.; Kurtz, S. M.; Rimnac, C. M.; Edidin, A. A. Biomaterials 2002, 23, 2329.

6. Arruda, E.; Boyce, M. J Mech Phys Solids 1993, 41, 389.

7. Arruda, E.; Boyce, M.; Jayachandran, R. Mech Mater 1995, 19, 193.
8. Wu, P. D.; van der Giessen, E. J Mech Phys Solids 1993, 41, 427.

9. Septanika, E. G.; Ernst, L. J. Mech Mater 1998, 30, 265.

10. Sweeney, J. Comput Theor Polym Sci 1999, 9, 27.

11. Ball, R.; Doi, M.; Edwards, S.; Warner, M. Polymer 1981, 22, 1010.

12. Edwards, S. F.; Vilgis, T. Polymer 1986, 27, 483.

13. Sweeney, J.; Naz, S. P.; Coates, D. J Appl Polym Sci 2009, 111, 1190.

14. Sweeney, J.; Spares, R.; Woodhead, M. Polym Eng Sci 2009, $49,1902$.

15. Sweeney, J.; Ward, I. M. J Mech Phys Solids 1996, 44, 1033.

16. Buckley, C. P.; Jones, D. Polymer 1995, 36, 3301.

17. Buckley, C. P.; Jones, D. Polymer 1996, 37, 2403.

18. Dooling, P. J.; Buckley, C. P.; Rostami, S.; Zahlan, N. Polymer 2002, 43, 2451.

19. Gorlier, E.; Agassant, J.-F.; Haudin, J.-M.; Billon, N. Plast Rubber Compos 2001, 30, 48.

20. Sweeney, J.; Ward, I. M. J Rheol 1995, 39, 861.

21. Lai, D.; Yakimets, I.; Guignon, M. Mater Sci Eng A 2005, 405, 266.

22. Drozdov, A.; Agarwal, S.; Gupta, R. Comp Mater Sci 2004, 29, 195.

23. Drozdov, A. Compos Sci Technol 2006, 66, 2648.

24. Drozdov, A. D.; Christiansen, J. de C. Int J Fracture 2009, 159, 63.

25. Drozdov, A. D.; Christiansen, J. de C. Int J Solids Struct 2008, 45,4274 .

26. Drozdov, A. D.; J. Christiansen, J. Int J Solids Struct 2009, 46, 2298.

27. Richeton, J.; Ahzi, S.; Vecchio, K. S.; Jiang, F. C.; Makradi, A. Int J Solids Struct 2007, 44, 7938.

28. Richeton, J.; Ahzi, S.; Daridon, L.; Rémond, Y. Polymer 2005, $46,6035$.

29. Septanika, E. G.; Ernst, L. J Mech Mater 1998, 30, 253.

30. Septanika, E. G.; Ernst, L. J. Mech Mater 1998, 30, 265.

31. Baquet, E. viwe hyper elastic thermo mechanical modeling for the behavior of a semi-crystallive polymer; app. to PA 66 . PhD thesis, Mines-ParisTech, 2011 (to be defended). 